Supporting Information

Formation of a Photoelectrochemical Z-Scheme Structure with Inorganic/Organic Hybrid Materials for Evaluation of Receptor Protein Expression on the Membrane of Cancer Cells

Zizheng Wang, ${ }^{\#,+}$ Jing Li,\#,+ Wenwen Tu, ${ }^{\dagger}$ Huaisheng Wang, ${ }^{\ddagger}$ Zhaoyin Wang ${ }^{*}+$ and Zhihui Dai*,

$\dagger$ Jiangsu Collaborative Innovation Center of Biomedical Functional Materials and Jiangsu Key Laboratory of Biofunctional Materials, School of Chemistry and Materials Science, Nanjing Normal University, Nanjing, 210023, P. R. China.

\$ School of Chemistry and Chemical Engineering, Liaocheng University, Liaocheng, 252059, P. R. China.

*Tel./Fax: +86-25-85891051. E-mail: daizhihuii@njnu.edu.cn; inzo@163.com.

\title{
List of contents:
}

1. Experimental section.

2. Solubility of AZIS QDs and L-Cys AZIS QDs (Figure S1)

3. Optimization of the detection conditions (Figure S2).

4. Cytotoxicity of L-Cys AZIS QDs/FePc hybrid materials (Figure S3).

5. Comparison of different PEC cytosensors (Table S1). 
6. Related references for supporting information (Ref. S1 to S7). 


\section{Experimental section}

\section{Materials and reagents}

Silver nitrate $\left(\mathrm{AgNO}_{3}\right)$, thioacetamide $(\mathrm{TAA})$, zinc acetate $\left(\mathrm{Zn}(\mathrm{OAc})_{2} \cdot 2 \mathrm{H}_{2} \mathrm{O}\right)$, indium nitrate $\left(\ln \left(\mathrm{NO}_{3}\right)_{3} \cdot 4.5 \mathrm{H}_{2} \mathrm{O}\right)$ ascorbic acid $(\mathrm{AA})$ and sodium hydroxide $(\mathrm{NaOH})$ were purchased from Sinopharm Chemical Reagent Co. Ltd. Tris (hydroxymethyl) aminomethane (Tris) was provided by Alfa Aesar. Iron phthalocyanine (FePc), L-Cysteine (L-Cys), 1-Ethyl-3-[(3-dimethylamino)-propyl)] carbodiimide (EDC), N-hydroxysuccinimide (NHS) and bovine serum albumin (BSA) were purchased from Sigma-Aldrich. Methyl thiazolyl tetrazolium (MTT) and hyaluronic acid (HA) were obtained from Shanghai Sangon Biotechnology Co., Ltd. Indium tin oxide (ITO) glasses were purchased from Kondrk Co., Ltd. All the other chemicals were of analytical grade. Ultrapure water $(\geqslant 18 \mathrm{M} \Omega \mathrm{cm})$ was used throughout all experiments. Tris- $\mathrm{HCl}$ buffer solution $(0.01 \mathrm{M}, \mathrm{pH} 7.4)$ containing $0.1 \mathrm{M} \mathrm{NaCl}$ and $0.05 \mathrm{M} \mathrm{KCl}$ served as the washing buffer solution.

\section{Apparatus}

Transmission electron microscope (TEM) images were recorded on transmission electron microscope (H-7650, Hitachi, Japan). Fourier transform infrared (FT-IR) spectra were recorded on FT-IR spectrometer (Tensor 27, Bruker, Germany). The X-ray powder diffraction (XRD) pattern was performed with a diffractometer (D/Max 2500 VL/PC, Japan). The spectrophotometer (Cary 60, Agilent, USA) and Fluoromax-4 spectrometer (Horiba, Japan) were applied to characterize the ultraviolet-visible (UV-vis) and photoluminescence (PL) spectra of the samples respectively. The Autolab PGSTAT302N (Metrohm, Netherlands) electrochemical workstation was used for electrochemical impedance spectroscopy (EIS) measurements. The KRUSS (Model DSA30S, Germany) was used for contact angles measurements. Photoelectrochemical (PEC) tests were implemented on Zahner PEC workstation (Zahner, Germany). Three-electrode system was used for all experimental measurements at room temperature, in which modified ITO electrode, platinum wire 
and $\mathrm{Ag} / \mathrm{AgCl}$ electrode were used as working electrode, opposite electrode and reference electrode, respectively. 
2. Solubility of AZIS QDs and L-Cys AZIS QDs (Figure S1)

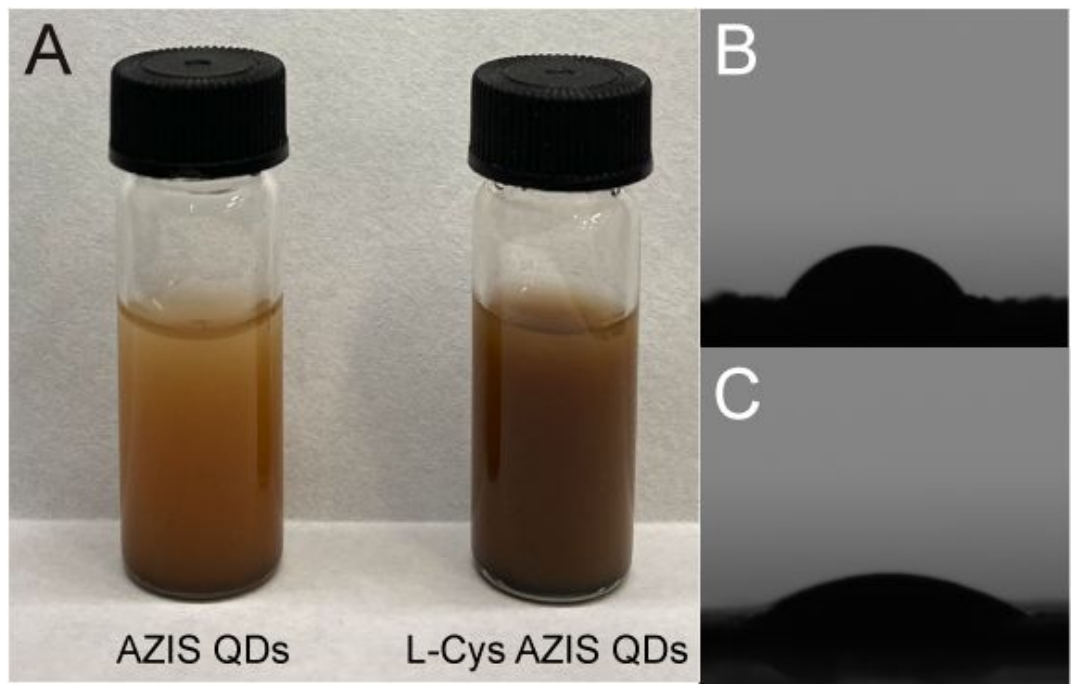

Figure S1. (A) Picture of AZIS QDs and L-Cys AZIS QDs in water. Contact angles of glass slide with (B) AZIS QDs and (C) L-Cys AZIS QDs. 
3. Optimization of the detection conditions
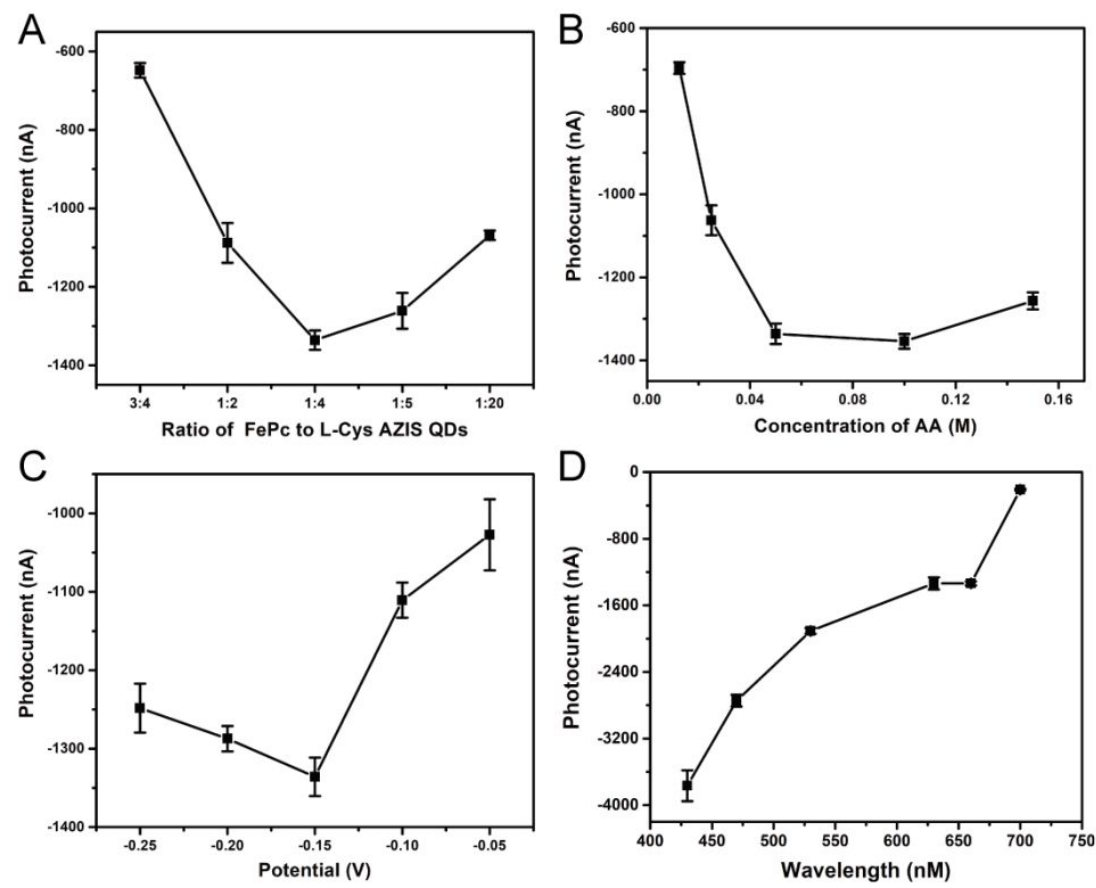

Figure S2. Optimization of PEC detection conditions: (A) ratio of FePc to L-Cys AZIS QDs, (B) concentration of AA, (C) bias voltage, and (D) excitation wavelength. 
4. Cytotoxicity of L-Cys AZIS QDs/FePc hybrid materials

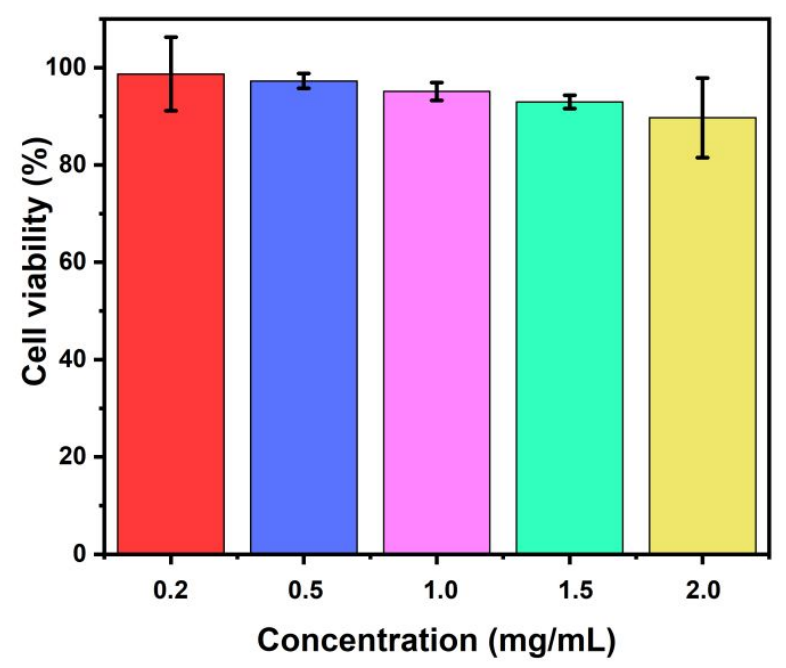

Figure S3. Cell viability of A549 cells after treatment with different concentrations of L-Cys AZIS QDs/FePc hybrid materials. 


\section{Comparison of different PEC cytosensors}

Table S1. Comparison of analytical performances of different PEC cytosensors

\begin{tabular}{llcc}
\hline Cell lines & $\begin{array}{l}\text { Linear range } \\
(\text { cell/mL })\end{array}$ & $\begin{array}{c}\text { Detection limit } \\
(\text { cell } / \mathrm{mL})\end{array}$ & References \\
\hline CCRF-CEM cells & $1.5 \times 10^{2}-3 \times 10^{5}$ & 16 & $\mathrm{~S} 1$ \\
CCRF-CEM cells & $2 \times 10^{1}-2 \times 10^{4}$ & 20 & $\mathrm{~S} 2$ \\
Apoptotic cells & $1 \times 10^{3}-5 \times 10^{7}$ & 158 & $\mathrm{~S} 3$ \\
Hela cells & $1 \times 10^{2}-1 \times 10^{6}$ & 34 & $\mathrm{~S} 4$ \\
MCF-7 cells & $1 \times 10^{3}-1 \times 10^{5}$ & 400 & $\mathrm{~S} 5$ \\
MCF-7 cells & $5 \times 10^{2}-5 \times 10^{6}$ & 198 & S6 \\
SKBR-3 cells & $1 \times 10^{2}-5 \times 10^{5}$ & 24 & S7 \\
A549 cells & $2 \times 10^{2}-4.5 \times 10^{6}$ & 15 & This work \\
\hline
\end{tabular}




\section{References}

S1. Li, J.; Lin, X. F.; Zhang, Z. Y.; Tu, W. W.; Dai, Z. H. Red Light-Driven Photoelectrochemical Biosensing for Ultrasensitive and Scatheless Assay of Tumor Cells Based on Hypotoxic AgInS 2 Nanoparticles. Biosens. Bioelectron. 2019, 126, 332-338.

S2. Wu, R.; Fan, G.-C.; Jiang, L.-P.; Zhu, J.-J. Peptide-Based Photoelectrochemical Cytosensor Using a Hollow $\mathrm{TiO}_{2} / \mathrm{EG} / \mathrm{ZnIn}_{2} \mathrm{~S}_{4}$ Cosensitized Structure for Ultrasensitive Detection of Early Apoptotic Cells and Drug Evaluation. ACS Appl. Mater. Interfaces 2018, 10, 4429-4438.

S3. Fan, G.-C.; Li, Z. M.; Lu, Y. W.; Ma, L. Z.; Zhao, H.; Luo, X. L. Robust Photoelectrochemical Cytosensor in Biological Media Using Antifouling Property of Zwitterionic Peptide. Sens. Actuators, B 2019, 299, 126996.

S4. Wang, K. W.; Zhang, R. H.; Sun, N.; Li, X. P.; Wang, J.; Cao, Y.; Pei, R. J. Near-Infrared Light-Driven Photoelectrochemical Aptasensor Based on the Upconversion Nanoparticles and $\mathrm{TiO}_{2} / \mathrm{CdTe}$ Heterostructure for Detection of Cancer Cells. ACS App. Mater. Interfaces 2016, 8, 25834-25839.

S5. Yang, H. M.; Zhang, Y.; Zhang, L. N.; Cui, K.; Ge, S. G.; Huang, J. D.; Yu, J. H. Stackable Lab-on-Paper Device with All-in-One Au Electrode for High-Efficiency Photoelectrochemical Cyto-Sensing. Anal. Chem. 2018, 90, 7212-7220.

S6. Pang, X. H.; Cui, C.; Su, M. H.; Wang, Y. G.; Wei, Q.; Tan, W. H. Construction of Self-Powered Cytosensing Device Based on ZnO Nanodisks@g- $\mathrm{C}_{3} \mathrm{~N}_{4}$ Quantum Dots and Application in the Detection of CCRF-CEM Cells. Nano Energy 2018, 46, 101-109.

S7. Liu, S. S.; He, P.; Hussain, S.; Lu, H.; Zhou, X.; Lv, F. T.; Liu, L. B.; Dai, Z. H.; Wang, S. Conjugated Polymer-Based Photoelectrochemical Cytosensor with Turn-On Enable Signal for Sensitive Cell Detection. ACS Appl. Mater. Interfaces 2018, 10, 6618-6623. 